\title{
Similarity of Rectangles: An Analysis of Subjective Dimensions ${ }^{1}$
}

\author{
David H. KRANTZ \\ University of Michigan, Ann Arbor, Michigan 48104
}

AND

Amos Tversky

Hebrew University, Jerusalem, Israel

\begin{abstract}
Two defining properties of psychological dimensions (intradimensional subtractivity and interdimensional additivity) are introduced and their consequences, formulated in terms of an ordinal dissimilarity scale, are derived. These consequences are investigated using dissimilarity judgments between rectangles to determine which of two alternative dimensional structures area $(A)$ and shape $(S)$, or width $(W)$ and height $(H)$, satisfies additivity and/or subtractivity. The results show that neither dimensional structure is acceptable, although $A \times S$ provides a better account for the data of most Ss than does $W \times H$. Tests of relative straightness show that $A$ is the least "curved" of the four attributes. Methodological and substantive implications of the study are discussed.
\end{abstract}

Much work in psychology is based on the assumption that stimuli (e.g., color patches, words, geometric figures) are perceived and evaluated in a dimensionally organized fashion. In speaking of hue, saturation, and brightness as dimensions of color spacc, or of potency as a dimension of scmantic space, it is typically assumed that these dimensions serve as organizing principles in the perception and the evaluation of colors or words. Despite its intuitive appeal, the precise content of this assumption is far from clear, particularly because the notion of psychological dimension is used in the literature in several different senses. How, then, does one test the hypothesis that a particular variable (specified physically or inferred via some model) acts like a subjective dimension? To answer this question, one needs a theory that specifies the formal properties of psychological dimensions-properties that are

1 This research was supported in part by NSF Grants GB-4947, GB-8181, and GB-6782 to the University of Michigan. We thank Maya Bar-Hillel and Phipps Arabie for their assistance in data analyses and S. David Kriska for helpful criticism.

Copyright (C) 1975 by Academic Press, Inc. 
empirically testable and that can be used to diagnose whether a particular variable can be properly regarded as a psychological dimension.

The theoretical study of the foundations of multidimensional-scaling models of similarity data (Beals, Krantz, \& Tversky, 1968; Tversky \& Krantz, 1970) has isolated two basic properties of subjective dimensions: interdimensional additivity (1), and intradimensional subtractivity (2). To formulate these properties, let $x=\left(x_{1}, \ldots, x_{n}\right)$, $y=\left(y_{1}, \ldots, y_{n}\right)$ denote two stimuli that vary along some $n$ attributes, and let $\delta(x, y)$ be an ordinal-scale measure of the dissimilarity between $x$ and $y$. It is assumed that for any $x \neq y$

$$
\delta(x, y)=\delta(y, x)>\delta(x, x)=\delta(y, y) .
$$

That is, $\delta$ is an ordinal distance measure: it is symmetric in its two arguments, and it is minimal between a point and itself. Using this index, interdimensional additivity asserts that the contributions of different dimensions to the overall dissimilarity between $x$ and $y$ are combined so that $\delta(x, y)$ is monotonically related to the sum of terms $\phi_{i}\left(x_{i}, y_{i}\right)$, where $x_{i}, y_{i}$ are the (nominal-scale) values of $x$ and $y$ on the $i$ th dimension, and $\phi_{i}$ is a symmetric real-valued function taking the value 0 when $x_{i}=y_{i}$ and positive values for $x_{i} \neq y_{i}$. That is,

$$
\delta(x, y)=F\left[\sum_{i=1}^{n} \phi_{i}\left(x_{i}, y_{i}\right)\right],
$$

where $\boldsymbol{F}$ is a strictly increasing function of one variable. Intradimensional subtractivity says that the contribution of any one dimension to dissimilarity depends on the absolute difference of rescaled (interval-scale) measures on that dimension, i.e.,

$$
\delta(x, y)=F\left[\left|f_{1}\left(x_{1}\right)-f_{1}\left(y_{1}\right)\right|, \ldots,\left|f_{n}\left(x_{n}\right)-f_{n}\left(y_{n}\right)\right|\right],
$$

where $F$ is a function of $n$ variables, strictly increasing in each variable taken separately, and the $f$ 's are "psychophysical functions" that rescale the nominal variables $x_{1}$, $x_{2}, \ldots, x_{n}$ to interval-scale variables $f_{1}\left(x_{1}\right), f_{2}\left(x_{2}\right), \ldots, f_{n}\left(x_{n}\right)$.

The above equations describe two natural algebraic properties of subjective dimensions: additivity across dimensions, and subtractivity along each dimension. Both properties are assumed in practically all multidimensional-scaling models of similarity data. They can be regarded, therefore, as defining properties for subjective dimensions; that is, subjective dimensions are expected to satisfy at least one of these properties.

The purpose of the present study is to demonstrate in detail the testing of (1) and (2), on the basis of ordinal dissimilarity judgments of individual subjects, in order to diagnose subjective dimensions. For this purpose we consider four physical properties of rectangles, width $(W)$, height $(H)$, area $(A=W H)$, and shape $(S=W / H)$, as possible candidates for subjective dimensions. In particular, we compare two alternative dimensional (product) structures, $W \times H$ vs. $A \times S$, to determine which, if either, satisfies additivity and/or subtractivity. 


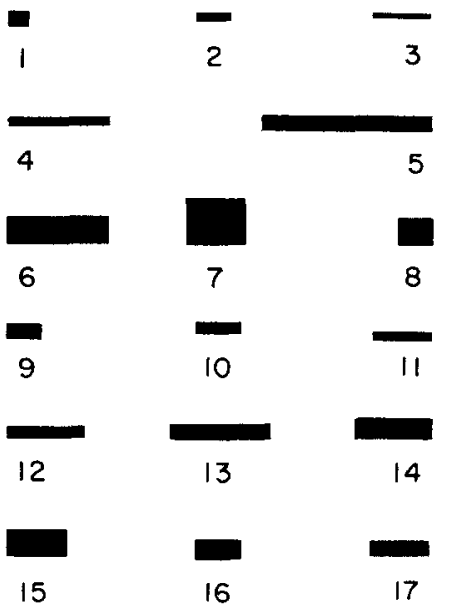

FIg. 1. Photograph of the 17 rectangles used as stimuli, with corresponding numbers.

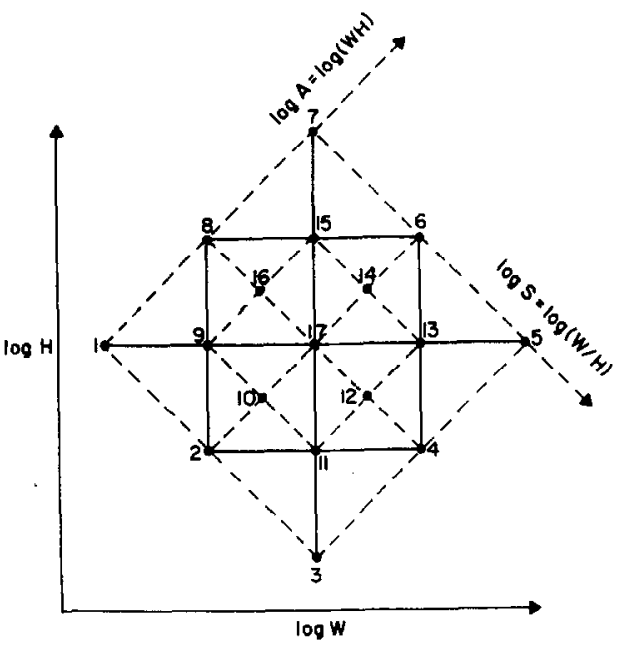

FIG. 2. The 17 rectangles of Fig. 1 are plotled in $\log$ width $\times \log$ height orthogonal coordinates. The grid of $\pm 45^{\circ}$ lines constitutes log area $\times \log$ shape orthogonal coordinates. Note that the 17 rectangles are a symmetric subset of a $5 \times 5 A \times S$ factorial design. The value of $S=W / H$ of the central stimulus (17) is 3.72 and the ratio between the adjacent levels along the $W$ and $H$ factors is 1.30. For example, the ratio of the heights of 16 to 9 equals the ratio of the heights of 16 to 17 which equals 1.30 . 
The stimuli were black rectangles on a light background. The 17 rectangles used are shown in Fig. 1. In Fig. 2, each rectangle is represented as a point in a $W \times H$ coordinate system. The levels of $W$ and $H$ are equally spaced on a logarithmic scale (see caption of Fig. 2 for numerical values). This spacing results in a simultaneous logarithmically spaced $A \times S$ factorial design, shown by the broken lines at $45^{\circ}$ to the $W, H$ axes in Fig. 2.

The physical equivalence classes (lines of constant $W, H, A$, or $S$ in Fig. 2) correspond to psychological equivalence classes, to a high degree of approximation. There is little if any illusion in perceived $W, H$, or $S$ : two rectangles of different area that are geometrically similar (equal $S$ ) look to be the same shape. A substantial area illusion does exist: tall rectangles look bigger than shorter one of equal area. To avoid this, we chose all our rectangles to be squat: the largest $H$ value was smaller than the smallest $W$ value. For physical $A$-equivalence classes, no systematic variation of perceived area was observed, within this range of squat shapes.

Our stimulus set thus gives us the larger part of a $5 \times 5$ factorial design in perceived $A \times S$, and, simultaneously, a $3 \times 3$ and a $2 \times 2$ factorial design in perceived $W \times H$ (with 4 outlying points, stimuli Nos. 1, 3, 5, 7). Using these stimuli, we collected replicated dissimilarity judgments for pairs of rectangles and tested interdimensional additivity (1) for the $A \times S$ and the $W \times H$ factors, and intradimensional subtractivity (2) for "lines" of constant $A, S, W$, or $H$.

Additional tests of these properties, using schematic faces as test stimuli, are described in Tversky \& Krantz (1969); for another test of (1) and (2) using rectangles, see Wender (1971).

\section{THEORY}

\section{Notation}

Throughout the remaining sections, we refer repeatedly to Fig. 2. Readers will find the notation, predictions, and discussion much easier to follow and to remember if the geometric language is followed through Fig. 2.

For convenience we refer to $A$ levels as $a, a^{\prime}, a^{\prime \prime}$, etc., instead of $x_{i}, y_{i}$, etc. The $A$ level of rectangle $i$ may also be denoted at times by $a_{i}$; hence, from Fig. 2, $a_{1}=a_{2}=$ $a_{3}, a_{9}=a_{10}=a_{11}$, etc. Similar notation is used for $S, W$, or $H$ levels.

The rectangle with area $a$ and shape $s$ will be denoted $a s$; thus, $i=a_{i} s_{i}=h_{i} w_{i}$.

It is also convenient to introduce a notation for pairs of $A$ levels, $S$ levels, etc. We refer to pairs of $A$ levels as $A$ intervals, and denote the four atomic $A$ intervals as $A_{1}, A_{2}, A_{3}, A_{4}$, proceeding from low area to high area. Thus, $A_{1}=\left(a_{2}, a_{10}\right)$, $A_{2}=\left(a_{9}, a_{16}\right)=\left(a_{10}, a_{17}\right)=\left(a_{11}, a_{12}\right)$, etc. Combinations of adjacent intervals are denoted by combined subscripts; e.g., $A_{12}=A_{1}+A_{2}=\left(a_{1}, a_{8}\right)=\left(a_{2}, a_{17}\right)=$ 
$\left(a_{3}, a_{4}\right)$, etc. The other combined $A$ intervals are $A_{23}, A_{34}, A_{123}, A_{234}$, and $A_{1234}=$ $\left(a_{1}, a_{7}\right)=\left(a_{2}, a_{6}\right)=\left(a_{3}, a_{5}\right)$. Similar notation is introduced for $S$ intervals, proceeding from least to most extreme shape: $S_{1}=\left(s_{8}, s_{16}\right), \ldots, S_{4}=\left(s_{12}, s_{4}\right)$, etc. Likewise, $H_{1}, \ldots, H_{1234}$, and $W_{1}, \ldots, W_{1234}$ refer to $H$ and $W$ intervals, with $H_{1}=$ $\left(h_{3}, h_{11}\right)$, etc. and $W_{1}=\left(w_{1}, w_{9}\right)$, etc.

Using this notation, pairs of stimuli can be given explicit $A \times S$ or $W \times H$ notations, e.g., $(1,2)=S_{12}=W_{1} H_{2} ;(9,13)=A_{23} S_{23}=W_{23}$, etc. Note that we use seven levels of $W$ and $H$, but introduce notation only for the four main intervals and their sums. Hence, not every pair has a $W \times H$ notation, e.g., $(10,9)$ although every pair has an $A \times S$ notation. Also note that as many as three different pairs of rectangles may share the same notation in $A \times S$ or $W \times H$ intervals, e.g., $W_{2}=(8,15)=$ $(9,17)=(2,11)$. To take another example, the notation $A_{12} S_{12}$ refers to both $(1,17)$ and $(2,8)$. These two pairs form the vertices of a square, 1, 2, 17, 8, in Fig. 2, with diagonals $(1,17)$ and $(2,8)$. Similarly, $A_{1234} S_{12}$ indicates the rectangle in Fig. 2 with vertices $1,2,6,7$, and diagonals $(1,6)$ and $(2,7)$.

\section{Interdimensional Additivity}

We first state the testable consequences of interdimensional additivity for the specific case of $A \times S$ dimensions. The theory for $W \times H$ dimensions is parallel and needs no separate statement.

The first two sets of predictions from Eq. (1) are actually derivable from a generalization of that equation. We rewrite Eq. (1) in the newly introduced notation as

$$
\delta\left(a s, a^{\prime} s^{\prime}\right)=F\left[\phi\left(a, a^{\prime}\right)+\psi\left(s, s^{\prime}\right)\right] .
$$

Its generalization, the equation of decomposability, is

$$
\delta\left(a s, a^{\prime} s^{\prime}\right)=F\left[\phi\left(a, a^{\prime}\right), \psi\left(s, s^{\prime}\right)\right] .
$$

In Eq. (3), $F$ becomes an arbitrary function of two variables, strictly increasing in each variable separately, rather than a strictly increasing function of their sum. In both Eqs. ( $\left(1^{\prime}\right)$ and (3), $\phi$ and $\psi$ are symmetric functions of the two nominal variables, taking the value of 0 for $a=a^{\prime}$ or $s=s^{\prime}$, and positive values otherwise. The additive combination rule of Eqs. (1) or (1') is replaced in Eq. (3) by some other combination rule, determined by the particular function $F$, but dissimilarity can still be decomposed into the contributions of two dimensions.

The two types of predictions based on Eq. (3) are called equality predictions and ordering predictions. The equality predictions are easily summarized in terms of the interval notation introduced above: any pairs designated by the same interval notation have equal dissimilarity. Thus, the three $A_{2}$ pairs, $(9,16),(10,17),(11,12)$, yield the prediction

$$
\delta(9,16)=\delta(10,17)=\delta(11,12)
$$


while the two $A_{12} S_{12}$ pairs, $(1,17)$ and $(2,8)$, yield the prediction

$$
\delta(1,17)=\delta(2,8) \text {. }
$$

The latter type of prediction is called a diagonal equality prediction. Inspection of Fig. 2 shows six triples of the former type for $A$, six for $S$, and six diagonal $A \times S$ predictions.

The ordering predictions may be summarized as follows: any ordering of several $A$ intervals is preserved by adding any one $S$ interval to each of them, and vice versa. For example, if $A_{2}>A_{1}$ (i.e., $\delta(10,17)>\delta(2,10)$ ), then $A_{2} S_{2}>A_{1} S_{2}$ and $A_{2} S_{3}>$ $A_{1} S_{3}$ (i.e., $\delta(9,17)>\delta(2,9)$ and $\delta(11,17)>\delta(2,11)$ ).

Equality Predictions. To show that equality predictions follow from Eq. (3), consider two diagonal pairs, $\left(a s, a^{\prime} s^{\prime}\right)$ and $\left(a s^{\prime}, a^{\prime} s\right)$. We have $\psi\left(s, s^{\prime}\right)=\psi\left(s^{\prime}, s\right)$ by symmetry. Hence

$$
\begin{aligned}
\delta\left(a s, a^{\prime} s^{\prime}\right) & =F\left[\phi\left(a, a^{\prime}\right), \psi\left(s, s^{\prime}\right)\right] \\
& =F\left[\phi\left(a, a^{\prime}\right), \psi\left(s^{\prime}, s\right)\right] \\
& =\delta\left(a s^{\prime}, a^{\prime} s\right) .
\end{aligned}
$$

A similar argument proves that $\delta\left(a s, a^{\prime} s\right)$ is independent of $s$, using the fact that $\psi(s, s)=0$, independent of $s$.

Ordering Predictions. To show that ordering predictions follow from Eq. (3), suppose that $A$ intervals $\left(a, a^{\prime}\right)$ and $\left(a^{\prime \prime}, a^{\prime \prime \prime}\right)$ are ordered in a certain way, e.g.,

$$
\delta\left(a s, a^{\prime} s\right)>\delta\left(a^{\prime \prime} s, a^{\prime \prime \prime} s\right)
$$

for some $s$ (hence, by equality, for every $s$ ). From the fact that $F$ is strictly increasing in the first variable, we infer that

$$
\phi\left(a, a^{\prime}\right)>\phi\left(a^{\prime \prime}, a^{\prime \prime \prime}\right) .
$$

Hence, for any common $S$ interval $\left(s^{\prime}, s^{\prime \prime}\right)$, we have (by monotonicity of $F$ in the first variable)

$$
\begin{gathered}
F\left[\phi\left(a, a^{\prime}\right), \psi\left(s^{\prime}, s^{\prime \prime}\right)\right]>F\left[\phi\left(a^{\prime \prime}, a^{\prime \prime \prime}\right), \psi\left(s^{\prime}, s^{\prime \prime}\right)\right], \text { or } \\
\delta\left(a s^{\prime}, a^{\prime} s^{\prime \prime}\right)>\delta\left(a^{\prime \prime} s^{\prime}, a^{\prime \prime \prime} s^{\prime \prime}\right), \text { as asserted. }
\end{gathered}
$$

The two types of predictions described above (equality and ordering) do not exhaust the testable consequences of Eq. $\left(1^{\prime}\right)$. Another class of predictions, which do not follow from decomposability (Eq. (3)) alone, are called cancellation predictions (Krantz, Luce, Suppes, \& Tversky, 1971). In this paper we do not test these predictions directly. Instead, we consider the pairs of rectangles as elements in an incomplete $11 \times 11$ factorial design whose factors are $A$ intervals and $S$ intervals. This design is shown in 
the matrix of Table 1. Each of the 92 pairs in our final design is entered in the appropriate cell, and each cell contains zero to three pairs. Pairs occupying the same cell should have equal dissimilarity (equality predictions). Thus, the ordinary equality predictions are represented in the first row and first column, where one of the dimensions has a zero interval, and the diagonal equality predictions are represented in the six cells having two pairs apiece.

TABLE 1

The 92 Experimental Pairs Arranged in an $A$ Interval $\times S$ Interval Matrix

\begin{tabular}{|c|c|c|c|c|c|c|c|c|c|c|c|}
\hline & $0_{s}$ & $S_{1}$ & $S_{\mathrm{a}}$ & $S_{3}$ & $S_{4}$ & $S_{12}$ & $S_{23}$ & $S_{34}$ & $S_{123}$ & $S_{234}$ & $S_{1234}$ \\
\hline $0_{A}$ & & 8,16 & $\begin{array}{r}9,10 \\
16,17 \\
14,15\end{array}$ & $\begin{array}{l}10,11 \\
12,17 \\
13,14\end{array}$ & 4,12 & $\begin{array}{l}1,2 \\
8,17 \\
6,7\end{array}$ & $\begin{array}{r}9,11 \\
12,16 \\
13,15\end{array}$ & $\begin{array}{l}2,3 \\
4,17 \\
5,6\end{array}$ & 8,12 & 4,16 & $\begin{array}{l}1,3 \\
4,8 \\
5,7\end{array}$ \\
\hline$A_{1}$ & 2,10 & 1,9 & 2,9 & 2,11 & 3,11 & & & & 1,11 & 3,9 & \\
\hline$A_{2}$ & $\begin{array}{r}9,16 \\
10,17 \\
11,12\end{array}$ & 8,9 & 9,17 & 11,17 & 4,11 & 8,10 & & 4,10 & & & \\
\hline$A_{3}$ & $\begin{array}{l}15,16 \\
14,17 \\
12,13\end{array}$ & 8,15 & 15,17 & 13,17 & 4,13 & 8,14 & & 4,14 & & & \\
\hline$A_{4}$ & 6,14 & 7,15 & 6,15 & 6,13 & 5,13 & & & & 7,13 & 5,15 & \\
\hline$A_{12}$ & $\begin{array}{l}1,8 \\
2,17 \\
3,4\end{array}$ & & 2,16 & 2,12 & & $\begin{array}{l}1,17 \\
2,8\end{array}$ & & $\begin{array}{l}2,4 \\
3,17\end{array}$ & & & \\
\hline$A_{23}$ & $\begin{array}{r}9,15 \\
10,14 \\
11,13\end{array}$ & & & & & & $\begin{array}{r}9,13 \\
11,15\end{array}$ & & & & \\
\hline$A_{34}$ & $\begin{array}{l}7,8 \\
6,17 \\
4,5\end{array}$ & & 6,16 & 6,12 & & $\begin{array}{l}6,8 \\
7,17\end{array}$ & & $\begin{array}{l}5,17 \\
4,6\end{array}$ & & & \\
\hline$A_{123}$ & 2,14 & 1,15 & & & 3,13 & & & & 1,13 & 3,15 & \\
\hline$\overline{A_{\mathrm{8} 34}}$ & 6,10 & 7,9 & & & 5,11 & & & & 7,11 & 5,9 & \\
\hline$A_{1234}$ & $\begin{array}{l}1,7 \\
2,6 \\
3,5\end{array}$ & & & & & & & & & & $\begin{array}{l}1,5 \\
3,7\end{array}$ \\
\hline
\end{tabular}


Additivity (Eq. $\left.\left(1^{\prime}\right)\right)$ is tested by trying to find scale values $\phi\left(A_{i}\right), \phi\left(A_{i j}\right)$, etc. and $\psi\left(S_{i}\right), \psi\left(S_{i j}\right)$, etc., such that the ordering of dissimilarities between cells is the same as the ordering of sums of scale values. The ordering predictions, implied by Fqs. (1') or (3), state that the orderings in any two columns coincide and the orderings in any two rows coincide. If equality and ordering hold, then additivity of the matrix is equivalent to the conjunction of all possible cancellation conditions (see Krantz, et al., 1971, Chapter 9). The actual test of additivity, in the face of noisy data, is discussed in the results section.

A similar matrix, for $W \times H$ dimension, is shown in Table 2. Since not every pair was assigned a $W \times H$ notation, the matrix contains only 62 of the 92 pairs. (Inclusion of the missing pairs would have complicated the analysis considerably by adding seven extra $W$ and seven extra $H$ intervals.)

TABLE 2

62 of the Experimental Pairs Arranged in a $W$ Interval $\times H$ Interval Matrix

\begin{tabular}{|c|c|c|c|c|c|c|c|c|c|c|c|}
\hline & $0_{H}$ & $H_{1}$ & $I_{2}$ & $I I_{3}$ & $I_{4}$ & $I_{12}$ & $I I_{23}$ & $I I_{34}$ & $I_{123}$ & $I I_{234}$ & $I I_{1234}$ \\
\hline $0_{W}$ & & 3,11 & $\begin{array}{c}2,9 \\
11,17 \\
4,13\end{array}$ & $\begin{array}{r}8,9 \\
15,17 \\
6,13\end{array}$ & 7,15 & 3,17 & $\begin{array}{c}2,8 \\
11,15 \\
4,6\end{array}$ & 7,17 & 3,15 & 7,11 & 3,7 \\
\hline$W_{1}$ & 1,9 & & 1,2 & 1,8 & & & & & & & \\
\hline$W_{2}$ & $\begin{array}{l}8,15 \\
9,17 \\
2,11\end{array}$ & 2,3 & $\begin{array}{l}2,17 \\
9,11\end{array}$ & $\begin{array}{l}9,15 \\
8,17\end{array}$ & 7,8 & 3,9 & & 7,9 & & & \\
\hline$W_{3}$ & $\begin{array}{r}6,15 \\
13,17 \\
4,11\end{array}$ & 3,4 & $\begin{array}{r}11,13 \\
4,17\end{array}$ & $\begin{array}{r}6,17 \\
13,15\end{array}$ & 6,7 & 3,13 & & 7,13 & & & \\
\hline$W_{4}$ & 5,13 & & 4,5 & 5,6 & & & & & & & \\
\hline$W_{12}$ & 1,17 & & 1,11 & 1,15 & & 1,3 & & 1,7 & & & \\
\hline$W_{23}$ & $\begin{array}{l}6,8 \\
9,13 \\
2,4\end{array}$ & & & & & & $\begin{array}{l}2,6 \\
4,8\end{array}$ & & & & \\
\hline$W_{34}$ & 5,17 & & 5,11 & 5,15 & & 3,5 & & 5,7 & & & \\
\hline$W_{123}$ & 1,13 & & & & & & & & & & \\
\hline$W_{234}$ & 5,9 & & & & & & & & & & \\
\hline$W_{1234}$ & 1,5 & & & & & & & & & & \\
\hline
\end{tabular}


The axiomatic analysis of interdimensional additivity (Tversky \& Krantz, 1970) has shown that, in the presence of some technical smoothness conditions, the properties expressed by the equality and ordering predictions are not only necessary, but sufficient, for decomposability (Eq. (3) with some function $F$ ), and the addition of one or two simple cancellation conditions guarantees interdimensional additivity. Hence, these predictions provide a thorough test of Eqs. (1') and (3).

\section{Intradimensional Subtractivity}

If we specialize Eq. (2) for $A \times S$ dimensions, it becomes

$$
\delta\left(a s, a^{\prime} s^{\prime}\right)=F\left[\left|f(a)-f\left(a^{\prime}\right)\right|, \quad\left|g(s)-g\left(s^{\prime}\right)\right|\right] .
$$

Clearly, this is a special case of Eq. (3), with

$$
\phi\left(a, a^{\prime}\right)=\left|f(a)-f\left(a^{\prime}\right)\right|, \quad \psi\left(s, s^{\prime}\right)=\left|g(s)-g\left(s^{\prime}\right)\right| .
$$

(Note that $\phi, \psi$, thus defined, are symmetric and take positive values for distinct variables if and only if $f$ and $g$ are one-to-one.) Thus, testing Eq. ( $\left.2^{\prime}\right)$ means testing Eq. (3), via equality and ordering predictions, plus testing that the functions $\phi, \psi$ have special forms as absolute differences. These latter tests can be carried out by fixing the value of one dimension, say, holding $s$ constant and varying $a$. 'l'hus, any tests which we develop here apply to any array of stimuli which are hypothesized to vary in only a single subjective dimension. For example, these tests apply to some cases of unidimensional unfolding (Coombs, 1964).

Consider, then, any set of stimuli (denoted $a, a^{\prime}, b, b^{\prime}, \ldots$ ) hypothesized to vary on only one dimension. We now derive predictions from the following model:

$$
\delta(a, b)=F[|f(a)-f(b)|] .
$$

If the stimulus array is really one-dimensional (i.e., Eq. $\left(2^{\prime \prime}\right)$ holds), then betweenness can be inferred from dissimilarity: we define $b$ to be between $a$ and $c$ (denoted $a|b| c$ ) if $\delta(a, c)$ is larger than both $\delta(a, b)$ and $\delta(b, c)$.

In most experimental applications, however, the betweenness relations are known a priori. In the present study, the a priori relations are inferred from Fig. 2. If these expectations are confirmed in the data, then the necessary betweenness axioms (Tversky \& Krantz, 1970) are automatically satisfied.

We use the betweenness relation to formulate further testable conditions. Suppose that $a|b| c$ and $a^{\prime}\left|b^{\prime}\right| c^{\prime}$. Then the additivity of adjacent absolute differences implies that if $\delta(a, b)>\delta\left(a^{\prime}, b^{\prime}\right)$ and $\delta(b, c) \geqslant\left(b^{\prime}, c^{\prime}\right)$, then $\delta(a, c)>\delta\left(a^{\prime}, c^{\prime}\right)$. We refer to these as monotonicity predictions (see Krantz et al., 1971, Chapter 4). Not all six 
stimuli need be distinct. A minimal test of this type is depicted in Fig. 3, using 4 stimuli, with $a=c^{\prime}, a^{\prime}=c$.

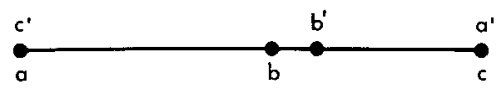

FIG. 3. Test of monotonicity based on four stimuli: if $\delta(a, b)>\delta\left(a^{\prime}, b^{\prime}\right)$, then $\delta\left(b^{\prime}, c^{\prime}\right)>\delta(b, c)$.

Thus, necessary conditions for a set of stimuli to be unidimensional are that the relation of betweenness is well-behaved, and that adjacent intervals are additive, in the sense of the monotonicity condition. Tversky and Krantz (1970) showed that, with continuity, these conditions are also sufficient. Closely related results were obtained by Suppes and Winet (1955).

Note that the additivity of adjacent intervals discussed above is much weaker than the additivity of dissimilarities,

$$
\delta(a, b)+\delta(b, c)=\delta(a, c) .
$$

The above equation, of course, is satisfied in the representation by absolute differences of scale valucs: if $a|b| c$, then

$$
|f(a)-f(b)|+|f(b)-f(c)|=|f(a)-f(c)| .
$$

But $\delta$ is only monotonically related to this additive measure, and so the observable constraints on $\delta$ are considerably weaker.

The final property to be tested is derived from the assumption of a metric with additive segments (Beals et al., 1968). This model postulates that any two stimuli form part of a unidimensional array, or additive segment. Practically all multidimensional-scalling models used in practice-the Euclidean and Minkowski power metrics -have this character, since distances are additive along all Euclidean straight lines in these models. If dissimilarity of rectangles is to be represented by a metric with additive segments, then one might well hypothesize that lines of constant $A, S, W$, or $H$ are particular additive segments.

To evaluate this hypothesis we use a condition akin to monotonicity, called relative straightness. Suppose, for example, that $a, b, c$ are three stimuli differing only in $A$ and that $w, x, y$ are three stimuli differing only in $W$. If the pattern of dissimilarities is such that $a|b| c, w|x| y, \delta(a, b)<\delta(w, x), \delta(b, c) \leqslant \delta(x, y)$, but $\delta(a, c) \geqslant \delta(w, y)$, then we say that $W$ is curved relative to $A$. Relative straightness coincides with monotonicity if the two triples lie along the same additive segment or dimension. Note that unlike monotonicity, relative straightness does not follow from Eq. (2) (intradimensional subtractivity).

If for example $A$ hehaves like an additive segment (with $S$ constant) but $W$ does not (with $H$ contant), then tests of relative straightness should show that $W$ is curved 
relative to $A$. If $A, S, W, H$ all behave like additive segments, then no one of them should be systematically curved relative to another. In addition, triples lying along these lines should be at least as straight as triples selected off these lines; e.g., referring to Fig. 2, the triple 2, 16, 6 should be curved relative to 2, 17, 6 .

An examination of relative straightness simplifies statistical testing by introducing a comparative standard. If monotonicity is violated in data, the question of sampling error is hard to deal with. But if the two tripies are drawn from different hypothetical dimensions, the violation is always directional, and one can observe whether there is a preponderance of such violations in one of the two directions.

\section{METHOD}

\section{Procedure}

Subjects $(S s)$ were presented a series of pairs of rectangles. Before the first session, they were read the following instructions:

In this experiment we will show you pairs of rectangles and we'll ask you to mark an $X$ in the appropriate cell on the scale from 1 to 20 , [answer booklet was before subject] according to the degree of dissimilarity between the rectangles.

For example: if the rectangles are almost identical, that is, the dissimilarity between them is very small, mark $X$ in a low-numbered cell. If the rectangles are very different from one another, mark $X$ in a high-numbered cell. In the same fashion, for all intermediate levels of dissimilarity between the rectangles, mark $X$ in an intermediate-numbered cell.

We are interested in your subjective impression of degree of dissimilarity. Different people are likely to have different impressions. Hence, there are no correct or incorrect answers. Simply look at the rectangles for a short time, and mark $X$ in the cell whose number appears to correspond to the degree of dissinilarity between the rectangles.

At the beginning of subsequent sessions, subjects were simply reminded that low numbers should correspond to low dissimilarity, high numbers to high dissimilarity. The words "similarity" and "difference" were scrupulously avoided, as was any mention of area, shape, height, width, or any other properties of rectangles.

Subjects were run in groups, usually eight or nine at a time, except when some $S s$ missed sessions and were run in smaller groups in make-up sessions.

Two sets of $S s$ were run. Group I consisted of nine seniors from a high school in Jerusalem, and Group II consisted of 8 undergraduates at the University of Michigan. (The instructions were written in Hebrew for Group I; a literal English translation, quoted above, was used for Group II.) Group I was run for scven scssions, Group II for ten sessions, each session lasting about forty-five minutes.

Stimuli were prepared by photographing rectangles cut out of black paper on a white background, at fixed reduction. From each, a positive 2 in. $\times 2$ in. slide was prepared. To present a pair of rectangles, the two were projected side-by-side on a white screen, 
using two matched slide projectors. (We did not succeed in making the light backgrounds completely identical, however.) Subjects were seated alongside or just behind the slide projector, so that there were only minor fluctuations in visual angle between sessions or between groups.

\section{Design}

A total of 92 pairs was presented. These were composed as follows: the 32 pairs connectcd by straight solid lincs in Fig. 2 [c.g., $(1,9),(1,17)$, etc.]; the 44 pairs connected by straight dotted lines in Fig. 2; the 8 pairs of form $(1,16),(16,7)$, $(7,14), \ldots ;$ and the 8 pairs of form $(1,15),(15,5),(7,13), \ldots$. These 92 pairs are shown in Table 1.

For Group I, the first session consisted of one complete run through the 92 pairs, in a random order, the results being discarded (by prior design, but unknown to the $S s$ ). The remaining six sessions consisted of one and one-half runs (138 trials), yielding a total of nine replications per pair. ${ }^{2}$ The 92 pairs were randomly divided into two blocks of 46 , denoted $A$ and $B$. Sessions consisted alternately of $A, B, A$ and $B, A, B$ sequences. The nine replications of block $A$ were run in nine different random orders, and the same was true of block $B$. A subject who missed a given session ran in a make-up session identical in block-sequence to the one he missed, but using new random orders within blocks. (This permitted us to use only two make-up sessions, $A, B, A$ and $B, A, B$, to accommodate all absentees.)

For Group II, the first session consisted of a complete run through the 92 pairs, in a new random order, the results again being discarded. The remaining nine sessions contained one presentation each of 70 pairs, plus two presentations of each of 22 pairs (consisting of the 22 diagonals of squares in Fig. 2), for a total of 114 trials. Each session used a new random order, except that the same pair was never presented twice successively within a session. Make-up sessions used new random orders. We thus obtained nine replications for 70 pairs and 18 replications for the other 22 pairs.

\section{RESULTS}

\section{Equality Predictions}

An examination of the mean dissimilarity ratings indicated a systematic departure from equality predictions. For example, $(1,3)$ was less dissimilar than $(5,7)$ for 15 out of $17 \mathrm{Ss}$, and $(4,8)$ was intermediate for 13 of those 15 , although all are $0_{A} \times S_{1234}$ pairs (see Fig. 2). As one goes from $(1,3)$ through $(4,8)$ to $(5,7)$, the level of the orthogonal $A$ dimension increases. This finding seemed to be fairly general: any fixed interval on one dimension tends to contribute more to dissimilarity as the level of the orthogonal dimension increases.

${ }^{2}$ Due to errors, a few pairs were replicated less than nine times for some $S s$ in Group I. 
To examine this possibility more closely, we calculated the differences, for each interval on one dimension, between mean dissimilarity ratings at successive levels of the orthogonal dimension. For example, for $S_{1234}$, we calculated the differences $\bar{\delta}(4,8)-\bar{\delta}(1,3)$ and $\bar{\delta}(5,7)-\bar{\delta}(4,8)$, where $\bar{\delta}(i, j)$ denotes the mean of 9 dissimilarity ratings of pair $(i, j)$. If these two differences are positive, the trend mentioned above is confirmed, while if they are negative, the opposite trend is indicated.

Table 3 presents the numbers and averages of the positive or negative differences of the above type, for $A \times S$ dimensions, for each of the $17 S s$. (The $S s$ from Groups I and II are numbered 1-9 and 10-17, respectively.) Under the null hypothesis (equality prediction), the number and average size of positive and negative differences should be equal.

TABLE 3

Equality Tests for $A \times S$ Dimensions ${ }^{a}$

\begin{tabular}{|c|c|c|c|c|c|c|c|c|}
\hline \multirow[b]{3}{*}{ Subject } & \multicolumn{4}{|c|}{ Area } & \multicolumn{4}{|c|}{ Shape } \\
\hline & \multicolumn{2}{|c|}{ Number } & \multicolumn{2}{|c|}{ Average } & \multicolumn{2}{|c|}{ Number } & \multicolumn{2}{|c|}{ Average } \\
\hline & + & - & + & - & + & - & + & - \\
\hline 1 & 10 & 2 & 1.04 & 0.42 & 12 & 0 & 1.30 & - \\
\hline 2 & 7 & 5 & 1.63 & 1.50 & 8 & 4 & 1.33 & 0.41 \\
\hline 3 & 7 & 5 & 0.56 & 0.56 & 8 & 4 & 0.85 & 0.72 \\
\hline 4 & 8 & 3 & 1.56 & 0.18 & 10 & 2 & 1.54 & 0.22 \\
\hline 5 & 6 & 4 & 0.72 & 1.45 & 11 & 1 & 0.73 & 0.66 \\
\hline 6 & 6 & 6 & 1.34 & 1.42 & 12 & 0 & 2.82 & - \\
\hline 7 & 8 & 4 & 1.22 & 0.94 & 10 & 2 & 1.96 & 0.78 \\
\hline 8 & 8 & 4 & 1.72 & 0.38 & 8 & 4 & 1.26 & 0.66 \\
\hline 9 & 7 & 5 & 0.92 & 0.85 & 12 & 0 & 0.97 & - \\
\hline 10 & 7 & 5 & 1.90 & 0.42 & 11 & 1 & 1.23 & 0.61 \\
\hline 11 & 11 & 1 & 2.86 & 1.78 & 10 & 2 & 1.14 & 0.42 \\
\hline 12 & 10 & 2 & 1.68 & 0.97 & 12 & 0 & 1.94 & - \\
\hline 13 & 3 & 9 & 1.39 & 0.76 & 4 & 6 & 0.66 & 0.92 \\
\hline 14 & 7 & 5 & 0.59 & 0.58 & 7 & 5 & 0.62 & 0.35 \\
\hline 15 & 8 & 3 & 0.98 & 1.24 & 10 & 1 & 1.17 & 0.99 \\
\hline 16 & 8 & 4 & 1.26 & 0.29 & 7 & 5 & 0.64 & 0.45 \\
\hline 17 & 6 & 6 & 0.65 & 1.50 & 8 & 2 & 2.95 & 0.42 \\
\hline Average & 7.47 & 4.29 & 1.29 & 0.89 & 9.41 & 2.29 & 1.36 & 0.54 \\
\hline
\end{tabular}

\footnotetext{
${ }^{a}$ Entries are the number and average of positive $(+)$ or negative $(-)$ differences between mean dissimilarities predicted to be equal, for $A$ pairs ( $S$ constant) and $S$ pairs ( $A$ constant). Positive differences indicate tendency for $A$ or $S$ interval to yield more dissimilarity as $S$ or $A$ level becomes more extreme or larger, respectively, while negative differences indicate reverse tendency.
} 
The group data (last row) show that the trend toward larger dissimilarity for a given $A$ interval as $S$ becomes more extreme is small but highly reliable $(p<.001$ based on a sign test alone), whilc the trend toward larger dissimilarity for a given $S$ interval at larger areas is very marked, and, of course, highly reliable $(p<.001)$. The same effect is observed in the data of individual $S$ s. If we compare, for each subject, the total number of positive and negative differences (adding together $A$ and $S$ intervals), then the equality hypothesis is violated in the hypothesized direction for all but $S 13$. Furthermore, despite the low power of the test, the equality hypothesis can he rejected at the .05 level, by a two-tailed sign test, for 10 out of $17 \mathrm{Ss}$.

TABLE 4

Equality Tests for $W \times H$ Dimensions ${ }^{a}$

\begin{tabular}{|c|c|c|c|c|c|c|c|c|}
\hline \multirow[b]{3}{*}{ Subject } & \multicolumn{4}{|c|}{ Width } & \multicolumn{4}{|c|}{ Height } \\
\hline & \multicolumn{2}{|c|}{ Number } & \multicolumn{2}{|c|}{ Average } & \multicolumn{2}{|c|}{ Number } & \multicolumn{2}{|c|}{ Average } \\
\hline & + & - & + & - & + & - & + & - \\
\hline 1 & 5 & 1 & 1.24 & 1.55 & 3 & 3 & 1.38 & 0.68 \\
\hline 2 & 4 & 2 & 1.58 & 0.78 & 2 & 4 & 3.10 & 1.49 \\
\hline 3 & 4 & 2 & 0.43 & 0.80 & 2 & 4 & 0.44 & 0.53 \\
\hline 4 & 4 & 2 & 0.34 & 0.38 & 2 & 4 & 1.26 & 0.92 \\
\hline 5 & 2 & 4 & 0.87 & 0.48 & 5 & 1 & 0.58 & 0.19 \\
\hline 6 & 3 & 3 & 1.63 & 1.20 & 4 & 2 & 1.37 & 2.28 \\
\hline 7 & 3 & 3 & 0.83 & 0.46 & 3 & 3 & 2.04 & 0.96 \\
\hline 8 & 1 & 5 & 1.03 & 0.63 & 4 & 0 & 1.47 & - \\
\hline 9 & 2 & 3 & 0.67 & 0.16 & 5 & 1 & 0.46 & 0.57 \\
\hline 10 & 3 & 3 & 1.17 & 0.96 & 5 & 1 & 1.93 & 1.55 \\
\hline 11 & 4 & 2 & 0.92 & 1.11 & 6 & 0 & 1.53 & - \\
\hline 12 & 2 & 4 & 1.06 & 1.28 & 5 & 1 & 1.42 & 1.11 \\
\hline 13 & 6 & 0 & 0.80 & - & 0 & 6 & - & 0.88 \\
\hline 14 & 2 & 3 & 0.39 & 0.48 & 4 & 2 & 0.50 & 0.80 \\
\hline 15 & 2 & 3 & 0.58 & 0.63 & 5 & 1 & 1.13 & 0.89 \\
\hline 16 & 1 & 4 & 1.22 & 0.56 & 3 & 3 & 0.55 & 0.39 \\
\hline 17 & 3 & 1 & 0.61 & 0.66 & 4 & 2 & 2.79 & 2.20 \\
\hline Average & 3.00 & 2.65 & 0.90 & 0.81 & 3.65 & 2.24 & 1.37 & 1.10 \\
\hline
\end{tabular}

${ }^{a}$ Entries are the same as Table 3 with $W$ and $H$ replacing $A$ and $S$, respectively.

Table 4 presents the same analyses for $W \times H$ dimensions. The group data for $H$ intervals show a small but reliable $(p<.05$, 2-tailed sign test, plus consistent difference between size of positive and negative deviations) tendency in the same 
direction that was observed for $A$ and $S$ intervals. The $W$ intervals show a slight tendency in the same direction, which is not statistically significant. The number of tests per $S$ for $W \times H$ dimensions is only half that for $A \times S$ (see Fig. 2), so that tests for individual $S s$ are not sufficiently powerful to justify any conclusions.

TABLE 5

Equality Tests for Diagonals in $A \times S$ Dimensions ${ }^{x}$

\begin{tabular}{|c|c|c|c|c|}
\hline \multirow[b]{2}{*}{ Subject } & \multicolumn{2}{|c|}{ Number } & \multicolumn{2}{|c|}{ Average } \\
\hline & + & - & + & - \\
\hline 1 & 2 & 4 & 1.24 & 0.55 \\
\hline 2 & 4 & 2 & 1.33 & 0.95 \\
\hline 3 & 1 & 5 & 0.22 & 0.45 \\
\hline 4 & 0 & 6 & - & 2.86 \\
\hline 5 & 3 & 3 & 1.34 & 0.62 \\
\hline 6 & 4 & 2 & 3.19 & 3.52 \\
\hline 7 & 4 & 2 & 2.55 & 0.55 \\
\hline 8 & 3 & 3 & 0.13 & 1.21 \\
\hline 9 & 3 & 3 & 0.45 & 0.45 \\
\hline 10 & 0 & 6 & - & 1.94 \\
\hline 11 & 4 & 2 & 2.32 & 1.36 \\
\hline 12 & 6 & 0 & 3.08 & - \\
\hline 13 & 1 & 5 & 0.65 & 0.75 \\
\hline 14 & 1 & 5 & 0.28 & 1.20 \\
\hline 15 & 6 & 0 & 0.90 & - \\
\hline 16 & 5 & 0 & 0.60 & - \\
\hline 17 & 5 & 1 & 1.43 & 0.83 \\
\hline
\end{tabular}

- Entries are the number and the average of positive $(+)$ or negative $(-)$ differences between mean dissimilarities (predicted to be equal) for the $W$ diagonal and the $H$ diagonal of $A \times S$ squares (Fig. 2). Positive differences indicate $W$ diagonal larger than $H$ diagonal. Data from Ss 10-17 are more reliable, based on double the number of observations (see text).

The results of diagonal equality predictions are summarized in Table 5 (diagonals of $A \times S$ design) and Table 6 (diagonals of $W \times H$ design). In Table 5, positive differences refer to unequal diagonals where the diagonal that is a $W$ interval is larger than the $H$ diagonal; in Table 6, positive differences refer to $S$ diagonal larger than $A$ diagonal. (Recall that the individual mean dissimilarities for Group II subjects are based on 18 replications per pair, for these diagonal pairs, and so are more reliable than any other individual means we report.) 
TABLE 6

Equality Tests for Diagonals in $W \times H$ Dimensions $^{a}$

\begin{tabular}{cccccc}
\hline & \multicolumn{2}{c}{ Number } & & \multicolumn{2}{c}{ Average } \\
\cline { 2 - 3 } \cline { 5 - 5 } Subject & + & - & & + & - \\
\hline 1 & 2 & 3 & & 0.34 & 1.03 \\
2 & 1 & 3 & & 1.00 & 2.26 \\
3 & 5 & 0 & & 5.01 & - \\
4 & 5 & 0 & & 5.68 & - \\
5 & 0 & 5 & & - & 4.82 \\
6 & 2 & 3 & & 0.84 & 0.78 \\
7 & 4 & 1 & & 1.54 & 0.55 \\
8 & 5 & 0 & & 4.85 & - \\
9 & 5 & 0 & & 1.89 & - \\
10 & 4 & 1 & & 1.88 & 0.12 \\
11 & 5 & 0 & & 5.05 & - \\
12 & 4 & 1 & & 1.05 & 0.50 \\
13 & 5 & 0 & & 2.01 & - \\
14 & 0 & 5 & - & 8.88 \\
15 & 4 & 1 & 1.35 & 1.55 \\
16 & 0 & 5 & - & 7.45 \\
17 & 2 & 3 & 1.84 & 2.21 \\
\hline
\end{tabular}

a Same as 'Table 5, with $A$ and $H$ interchanged and $W$ and $S$ interchanged. (Positive differences indicate tendency to perceive $S$ intervals in Fig. 2 design as more dissimilar than $A$ intervals.)

For Group I (Ss 1-9) the $A \times S$ diagonals tend not to depart from equality in a systematic way ( $S 4$ is the main exception). For Group II ( $S s$ 10-17), with mean dissimilarities more reliable, there is a noticeable tendency to violate the equality prediction in one direction or another. Since the direction of the violations is quite different for different $S s$, we do not report or analyze the group data.

The data for the $W \times H$ diagonals also show departures from the equality prediction in one direction or another. More than one-half of the $S s$ produced the most extreme distribution of + and - (i.e., a $5: 0$ split). The probability of obtaining such a number of extreme patterns, under the diagonal equality hypothesis, is less than .0001 .

Note that diagonals in the $A \times S$ design correspond to $W$ or $H$ intervals and vice versa. Hence, systematic patterns of violations of diagonal equality predictions in one product structure not only cast doubt on its adequacy, but also provide information about the relative weight of the factors in the alternative product structure. For example, of the nine $S s$ producing a 5:0 pattern in the $W \times H$ design (see Table 6), six $(3,4,8,9,11,13)$ emphasized $S$ at the expense of $A$, while three $(5,14,16)$ did the opposite. This classification of subjects was borne out by an INDSCAL analysis (see below, Fig. 5). 


\section{Ordering Predictions.}

The finding of systematic departures from the equality predictions casts doubt on the validity of the ordering predictions. The essence of ordering predictions is that an ordering of $A$ intervals, say, is preserved by adding any common $S$ interval to them; and this implicitly assumes that a pair of $A$ levels defines the same interval regardless of the $S$ levels involved.

Consequently we test a modified version of the ordering prediction, which is more likely to obtain, and whose violations shed additional light on the appropriateness of $A \times S$ or $W \times H$ dimensions and on the weighting of $A$ or $S$ for subjects using $A \times S$ dimensions. The details are complicated and the reader may wish to skip this section. The conclusions regarding individuals' use of $A \times S$ dimensions are essentially the same as those inferred from the diagonal equality predictions. (Compare conclusions column of Table 8 with Table 6.)

The tested version of the ordering prediction is illustrated as follows. Suppose that $\delta(2,17)>\delta(6,17)$ (see Fig. 2). Instead of adding an arbitrary fixed $S$ interval to each of these two $A$ intervals, suppose we restrict ourselves to modifying the common stimulus, 17 , so as to introduce the same $S$ interval, e.g., by substituting one of the stimuli 8 or 4 for 17 . By substituting 8 for 17 , for example, we are adding the same $S$ interval defined by the pair $(8,17)$ to the two $A$ intervals defined by the pairs $(2,17)$ and $(17,6)$. One might expect that the addition of this common $S$ interval would preserve the inequality between the two $A$ intervals. Thus, one predicts that $\delta(2,8)>\delta(6,8)$ whenever $\delta(2,17)>\delta(6,17)$. There are many other such predictions, of course.

A natural alternative to these $A \times S$ ordering predictions, for subjects who use $W \times H$ dimensions and violate $A \times S$ diagonal equality, is that the added common $S$ interval will reverse or preserve the $A$ interval ordering depending on whether the resulting pairs are $(H, W)$ or $(W, H)$. For example, $(2,8)$ is an $H$ pair and $(6,8)$ a $W$ pair, while $(2,4)$ is a $W$ pair and $(4,6)$ and $H$ pair. Thus, a subject who uses $W \times H$ dimensions, with $W$ intervals weighted more heavily than corresponding $H$ intervals, would tend to reverse the inequality $\delta(2,17)>\delta(6,17)$ when 8 is substituted for 17 , yielding an $(H, W)$ pair of pairs, and to preserve the inequality and even increase the disparity when 4 is substituted for 17 , yielding a $(W, H)$ pair of pairs.

To test these alternatives, we considered all groups of three pairs of pairs, where one pair consisted of two adjacent $A$ intervals, a second pair consisted of a $W$ and an $H$ interval obtained from the first pair by changing its common stimulus along the $S$ axis, and the third pair consisted of the $H$ and $W$ intervals obtained by changing the common stimulus by an equal but opposite amount (on our log scale) along the $S$ axis. These tests are referred to as the $A$ ordering predictions. The ordering predictions for $S, W$, and $H$ are defined similarly. For each subject, there were five $A$ and five $S$ triples (of pairs of pairs) and six $W$ and six $H$ triples. 
For each triple, we computed the difference between the mean dissimilarities for each pair of pairs. The three differences were classified in four different ways:

(i) as violations or nonviolations, the latter if and only if all three had the same sign (since no triple had all three differences zero, a zero difference always led the triple to be classed as a violation);

(ii) according to the pattern of signs in the second and third pairs, with +- , for instance, denoting that the second pair yielded a + difference ( $W$ interval greater than $H$ interval) and the third pair yielded a - difference ( $W$ interval again greater than $H$ interval, since the movement in the opposite direction reverses the character of the two intervals), -+ denoting the reverse, etc. (note that,+--+ patterns are necessarily violations under (i), while,++-- may or may not be violations, depending on the sign of the first difference; zero differences are simply omitted from this classification);

(iii) according to the order of size of the differences in the second and third pairs, where $W>H$ denotes that the $(W, H)$ pair had a greater (algebraic) difference than the $(H, W)$ pair (note that any +- pattern, from (ii), is necessarily classified as $W>H$, but that some ++ and -- patterns are also classified as $W>H$; there were no cases of $W=H$ );

(iv) as close to or far from zero, depending on the average absolute magnitude of the three differences.

These classifications are summarized, for $A \times S$ tests, in Table 7. Column 1 gives the total $A$ and total $S$ violations (classification (i), with a possible total of five in each case); columns $2-5$ give the total numbers of,,+++--+ , and -- patterns for $A$ and $S$ triples (classification (ii)); column 6 gives the total numbers of $W>H$ (classification (iii) for $A$ and $S$ triples); and column 7 gives the average absolute differences (iv) for $A$ and $S$ triples. The last column summarizes some tentative conclusions based on the data.

Table 8 gives a similar summary for $W \times H$ tests.

It can be seen from Table 7 (cols. 2-3) that the total number of triples in which violations occur is quite large, amounting to $74 \%$ of the possible total. On the other hand, for seven of the $17 S s(3,5,8,9,14,15,16)$ the average absolute differences (col. 7) are small (less than or about 1 point on a 20-point scale), and the patterns of violations (cols. 2-5) and of size of difference (col. 6) are unsystematic. For these $S s$, the order of $A$ intervals and the order of $S$ intervals is essentially a null order: the logarithmic spacing on $A$ and $S$ yields approximately equal subjective differences. This equality of $A$ or of $S$ intervals is preserved when a common $S$ or $A$ interval is added. Thus, the ordering predictions for $A \times S$ are substantially confirmed for these seven $S s$. For two $S s(2,10)$, it is difficult to decide whether the ordering predictions are violated in a significant way. 


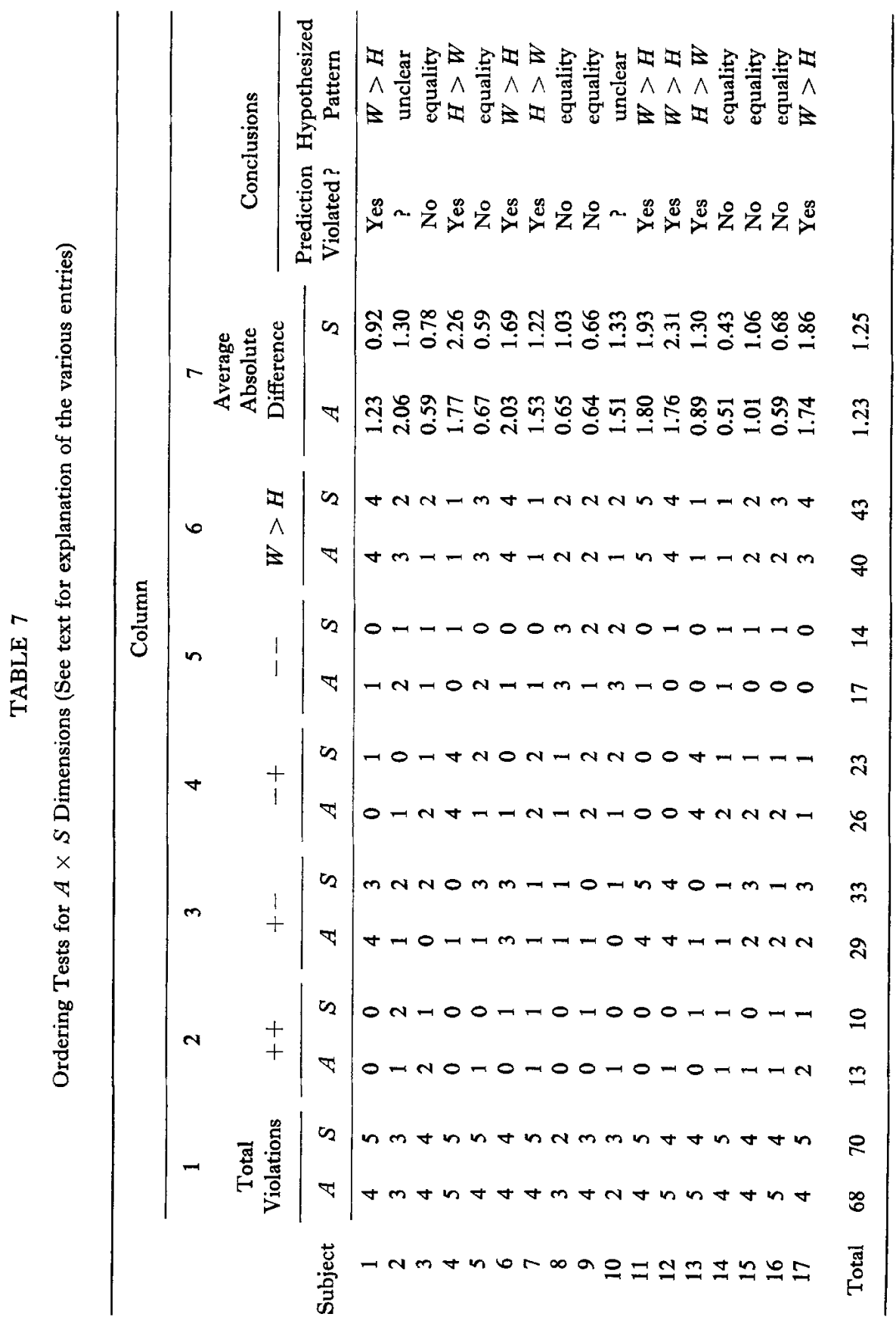




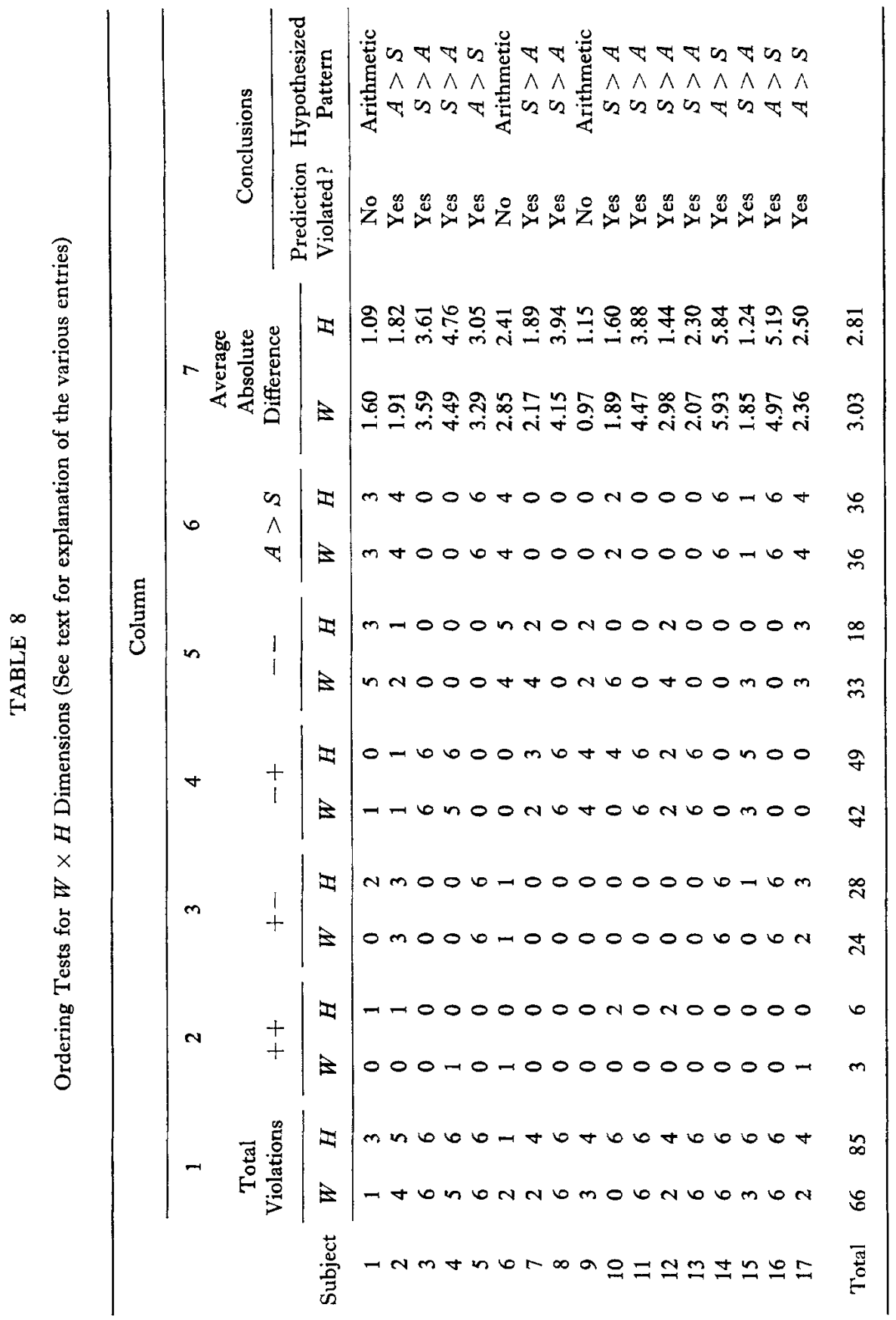


Eight $S s(1,4,6,7,11,12,13,17)$ clearly violate the ordering predictions, in a direction consistent with the use of $W \times H$ dimensions, with three $S s$ emphasizing $H$ and five emphasizing $W$. Nevertheless, the absolute differences are not very large for these $S$ s. It seems that the logarithmic spacing is not very far from equal spacing for all $S s$, with some $S s$ showing disturbance of this near-null ordering when an additional common interval on the orthogonal dimension is added and the rectangle pairs become $W$ and $H$ intervals. This disturbance is not a large effect, but it is sufficient to produce systematic violations in about half the $S s$.

A similar analysis of the data in Table 8 shows a very different picture for $W \times H$ dimensions. The overall percentage of violations is actually lower than for $A \times S$ dimensions- $-68 \%$ as opposed to $74 \%$-but the violations tend to be large and highly systematic. Three $S s(1,6,9)$ produce a relatively small number of violations, and their response pattern suggests a spacing of $W$ and $H$ which falls somewhere between a logarithmic and an arithmetic spacing indicated by "arithmetic" in the last column. For these $S s$, the ordering predictions cannot be readily rejected. The remaining 14 Ss seem to violate the $W \times H$ ordering prediction in a systematic fashion that is compatible with the use of $A \times S$ dimensions, with five $S s$ emphasizing $A$ and nine emphasizing $S$. Furthermore, eight of these $14 S s$ show average absolute differences that are considerably larger than any of those observed in the $A \times S$ tests reported in Table 7. In contrast to the latter tests, where even the most clearcut violations were small in magnitude, the violations observed in Table 8 are very large indeed.

We conclude, then, that logarithmic spacing is approximately equal for $W$ intervals and for $H$ intervals, though not as good as for $A$ and $S$ intervals; that the great majority of $S s$ (probably 14 out of 17 ) violate the $W \times H$ ordering predictions in favor of the alternative based on $A \times S$ dimensions, as opposed to eight such violations of the $A \times S$ ordering predictions in favor of the $W \times H$ alternative; and that some $W \times H$ violations are really large effects, while $A \times S$ violations are relatively smaller.

\section{Tests of Monotonicity and Relative Straightness}

As we have shown in the theoretical discussion, one can investigate the relative straightness, or curvedness, of subjective dimensions by testing monotonicity for two triples that lie on different dimensions. Table 9 presents the number of violations of monotonicity in which the row dimension appears curved relative to the column dimension, for all $S s$.

The main diagonal in each matrix shows the number of violations within each proposed dimension, i.e., the number of violations of monotonicity where both triples lie on the same dimension.

A summary of the data of Table 9 , for each one of the $S s$, is presented in Table 10 , where $\rightarrow$ denotes the relation of relative straightness (e.g., $A \rightarrow H, W$ means that both 
TABLE 9

Number of Monotonicity Violations in which the Row Dimension Appears Curved when Compared with the Column Dimension for each $S$

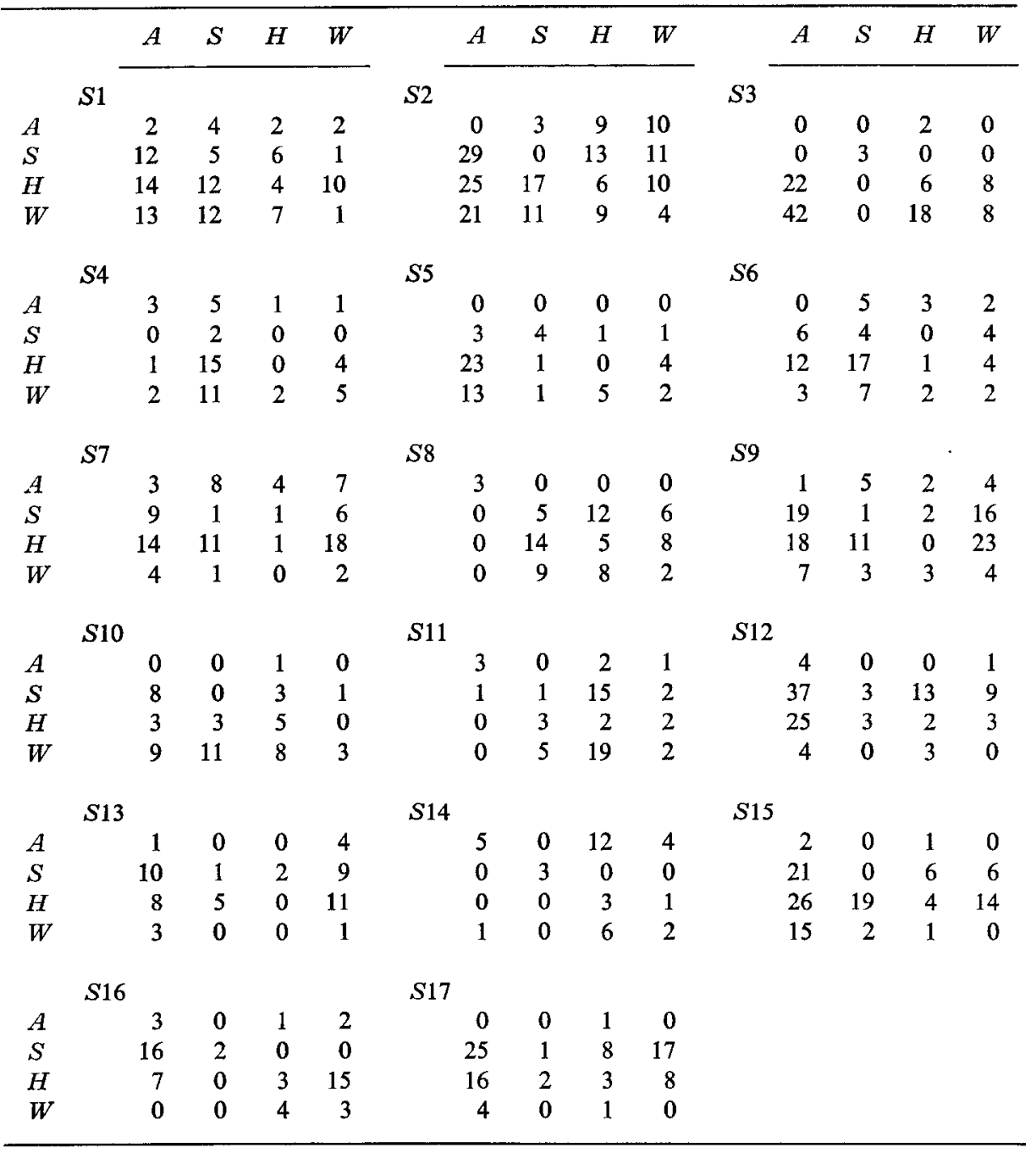

$H$ and $W$ appear curved relative to $A$ ). The symbol $>$ denotes dominance relation between dimensions, e.g., $S>A$ indicates that all $S$ intervals are greater than any $A$ interval. In this case, of course, the dominated dimension cannot appear curved relative to the dominating dimension. 
TABLE 10

Summary of the Relations of Relative Straightness for each $S$

\begin{tabular}{rll}
\hline Subject & $\rightarrow:$ is straighter than & $>:$ dominates \\
\hline 1 & $A \rightarrow S, H, W$ & \\
2 & $A \rightarrow S, H, W$ & $S>A, H, W$ \\
3 & $A \rightarrow H \rightarrow W$ & $S, H, W>A$ \\
4 & $S \rightarrow H, W$ & $A, H, W>S$ \\
5 & $A \rightarrow H, W$ & \\
6 & $A, S, W \rightarrow W$ & \\
7 & $W \rightarrow A, S \rightarrow H$ & \\
8 & & \\
9 & $A, W \rightarrow S \rightarrow H$ & \\
10 & $A \rightarrow S, H \rightarrow W$ & \\
11 & $H \rightarrow S \rightarrow W$ & \\
12 & $A \rightarrow W \rightarrow H \rightarrow S$ & \\
13 & $W, A \rightarrow S, H$ & \\
14 & $H \rightarrow W \rightarrow A$ & \\
15 & $A \rightarrow W \rightarrow S \rightarrow H$ & \\
16 & $W, A \rightarrow H \rightarrow S$ & \\
17 & $A \rightarrow W \rightarrow H \rightarrow S$ & \\
\hline
\end{tabular}

A further condensed summary of the tests of relative straightness is presented in Table 11, which displays the number of $S s$ for which the row dimension appears curved relative to the column dimension.

TABLE 11

Number of Ss for which the Row Dimension Appears Curved Relative to the Column Dimension (Based on Tables 9 and 10)

\begin{tabular}{lrrrr}
\hline & $A$ & $S$ & $H$ & $W$ \\
\hline$A$ & - & 0 & 0 & 1 \\
$S$ & 9 & - & 5 & 7 \\
$H$ & 13 & 5 & - & 10 \\
$W$ & 8 & 3 & 4 & - \\
\hline
\end{tabular}

Table 11 presents a very consistent picture: $A$ is clearly the "straightest" of the dimensions, $W$ is second, $S$ is third, and $H$ is the most curved dimension. 


\section{Additivity Analysis}

By considering pairs of rectangles as elements in an $A \times S$ factorial design (Table 1), or in a $W \times H$ factorial design ( $T$ able 2), interdimensional additivity can be tested by trying to assign scale values to the intervals of the respective (incomplete) design so that the ordering of their sums will coincide with the observed dissimilarity ordering. In order to keep the ratio of the number of parameters (i.e., scale values) to the number of data points (i.e., cell entries) reasonably low, we exclude from the present analysis the last row $\left(A_{1234}\right)$ and the last column $\left(S_{1234}\right)$ of Table 1 , as well as four rows

$$
\left(W_{23}, W_{123}, W_{234}, W_{1234}\right)
$$

and four columns $\left(H_{23}, H_{123}, I I_{234}, I I_{1234}\right)$ of Table 2 . Thus, we are left with a $10 \times 10$ design in $A \times S$ (with 59 nonempty cells), and a $7 \times 7$ design in $W \times H$ (with 36 nonempty cells).

In the presence of sampling error, some violations of additivity are inevitable and hence no perfect solution is available. To accommodate this state of affairs, an iterative grid search for finding a "hest fitting" additive solutions was devised.

Two sets of constraints, referred to as Model 1 and Model 2 were imposed on the solution. Model 1 merely requires that the scale values be compatible with the inclusion relation between intervals. That is, if one interval includes another, then the scale value of the former should be at least as large as that of the latter. For example,

$$
\phi\left(S_{1}\right) \leqslant \phi\left(S_{12}\right) \leqslant \phi\left(S_{123}\right),
$$

etc. Model 2, in effect, imposes intradimensional subtractivity, i.e., the scale value of an interval must equal the sum of the scale values of its subintervals. For example, $\phi\left(S_{12}\right)=\phi\left(S_{1}\right)+\phi\left(S_{2}\right), \phi\left(S_{123}\right)=\phi\left(S_{12}\right)+\phi\left(S_{3}\right)=\phi\left(S_{1}\right)+\phi\left(S_{23}\right)$, etc.

In additivity analysis, three scale values (interpreted as the origins of the two scales and their common unit of measurement) can be chusen arbitrarily. Thus, under Model 1 we estimate 17 and 11 parameters, respectively, for each (incomplete) $A \times S$ and $W \times H$ data matrix. Under Model 2, on the other hand, only 7 parameters are estimated for each of the designs; the other scale values are determined by the above equations.

For each $S$, a "best-fitting" additive solution (also referred to as prediction) was computed on the basis of the observed ordering of mean dissimilarities between the pairs of rectangles which correspond to the cells of the respective $(A \times S$ or $W \times H)$ design. In cases where several pairs are located in the same cell (see Tables 1 and 2), the overall mean dissimilarity was used to determine the rank order of the cell. For each design, separate solutions were calculated for each $S$ under each of the two models, yielding a total of four solutions per $S$. Each observed rank order was, then, compared with the rank order of the respective additive prediction, and the average absolute differences between the observed and the predicted orderings are presented in Table 12. 
TABLE 12

Average Absolute Differences Between Observed and Additive Orderings

\begin{tabular}{|c|c|c|c|c|}
\hline \multirow[b]{3}{*}{ Subject } & \multicolumn{4}{|c|}{ Design } \\
\hline & \multicolumn{2}{|c|}{$A \times S$} & \multicolumn{2}{|c|}{$W \times H$} \\
\hline & Model 1 & Model 2 & Model 1 & Model 2 \\
\hline 1 & 4.71 & 7.20 & 1.28 & 2.43 \\
\hline 2 & 7.46 & 8.37 & 4.12 & 4.38 \\
\hline 3 & 10.15 & 11.51 & 4.22 & 5.00 \\
\hline 4 & 6.63 & 6.15 & 4.52 & 3.75 \\
\hline 5 & 5.64 & 4.53 & 5.28 & 5.73 \\
\hline 6 & 5.98 & 5.94 & 1.83 & 2.00 \\
\hline 7 & 5.55 & 6.52 & 3.40 & 3.26 \\
\hline 8 & 6.03 & 7.18 & 6.08 & 6.50 \\
\hline 9 & 7.21 & 8.10 & 2.56 & 2.93 \\
\hline 10 & 4.59 & 6.46 & 2.47 & 3.69 \\
\hline 11 & 5.36 & 6.32 & 3.53 & 3.50 \\
\hline 12 & 7.08 & 6.78 & 3.71 & 4.47 \\
\hline 13 & 4.66 & 5.35 & 4.05 & 4.72 \\
\hline 14 & 4.28 & 4.86 & 6.68 & 6.64 \\
\hline 15 & 5.27 & 8.25 & 2.44 & 2.03 \\
\hline 16 & 3.15 & 3.43 & 5.97 & 6.38 \\
\hline 17 & 4.93 & 5.54 & 3.00 & 3.83 \\
\hline Average & 5.81 & 6.62 & 3.83 & 4.19 \\
\hline
\end{tabular}

Table 12 reveals substantial departures from interdimensional additivity. Unfortunately, there is no standard procedure for evaluating these statistics, since their expected value under the hypothesis of random ordering is not known. An upper bound for this value, however, is given by $(n+1)(n-1) / 3 n$ which is the expected average absolute deviation between two random rankings of $n$ objects. Under the random-ranking hypothesis, therefore, the reported statistics are expected to fall below 20 in the $A \times S$ design which contains 59 cells, and below 12 in the $A \times S$ design which contains 36 cells. Under interdimensional additivity, of course, all the entries for Model 1 should vanish. Thus, the data provide evidence against interdimensional additivity.

Since Model 2 is strictly more restrictive than Model 1, the average absolute differences should be greater for Model 2 than for Model 1. (The fact that for some Ss the deviations relative to Model 2 are slightly smaller than those relative to Model 1 is due to the fact that the criterion for the construction of the "best-fitting" additive 
solution $^{3}$ is not equivalent to the minimization of the average absolute difference between the observed and the predicted orderings.) In general, however, the differences between the two modcls are rclativcly small. Thus, the added constraints imposed by intradimensional subtractivity did not considerably reduce the quality of the fit, despite the large reduction in the number of free parameters.

\section{Multidimensional-Scaling Analysis}

In contrast to the present approach which investigates in detail the properties of some specifiable dimensions, the traditional multidimensional-scaling approach attempts to represent the stimuli as points in some multidimensional metric space and then to infer and interpret the psychological dimensions on the basis of the resulting configuration. It is instructive, therefore, to compare the approaches by applying multidimensional-scaling methods to the present data.

To obtain a representation of the rectangles in a two-dimensional Euclidean space, Kruskal's (1964) M-D-SCAL IV program was applied separately to the dissimilarity ordering of each $S$. The two-dimensional fit was acceptable for most $S s$; the average stress value (Kruskal's formula one ${ }^{4}$ ) was .10 . (The expected stress under the hypothesis of a random ordering is about .28; see Klahr, 1969.) For a more compact summary of the data, Carroll and Chang's (1970) INDSCAL program was employed using the resulting M-D-SCAL interpoint distances as input data. This program yields a common (Euclidean) group space, and a set of weights "assigned" by each $S$ to the dimensions of the space. (The weights, of course, are inferred via the model from the dissimilarities rather than estimated directly by the $S$.) Note that unlike the general Euclidean model, the INDSCAL program yields a preferred set of psychological dimensions.

The two-dimensional group space for the present data is shown in Fig. 4. Figure 5 represents each $S$ as a point in a space whose coordinates are the weights assigned by that $S$ to the two dimensions of the common space. Note that the points in Fig. 4 represent rectangles, while the points in Fig. 5 represent $S s$. The actual values of the weights are immaterial: only their ratio is of interest. However, the distance between each subject point and the origin in Fig. 5 increases with the goodness of fit of the model.

Inspection of Fig. 4 shows that the vertical and the horizontal dimensions, inferred via INDSCAL, correspond roughly to $A$ and $S$, respectively. (Compare Figs. 2 and 4.) It also shows that $A$ and $S$ are not orthogonal: the same $S$ interval appears larger, the higher the $A$ level. This was the strongest effect observed in Table 3 . Figures 4 and 5

\footnotetext{
${ }^{3}$ The solution criterion was the minimization of the sum of squared deviations between the observed and the predicted ranks.

${ }^{*}$ Both stress formulae one and two were tried; the former yielded a more sensible configuration with fewer iterations.
} 


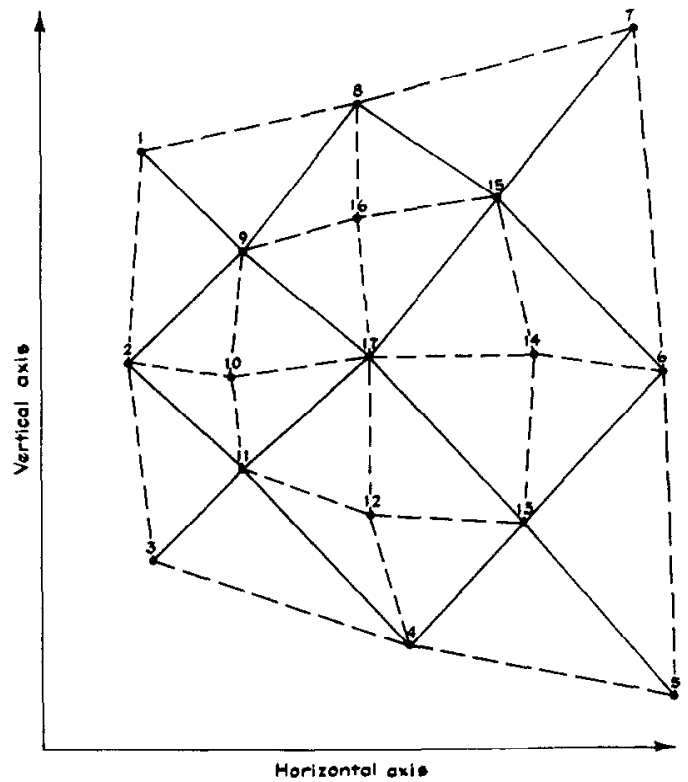

FIG. 4. The common space obtained by INDSCAL. Each point represents the location of the respective rectangle in the constructed space. The solid and broken lines represent, respectively, the $W \times H$ and the $A \times S$ designs (see Fig. 2).

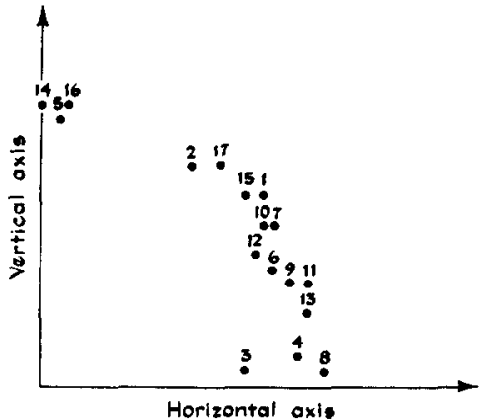

Fic. 5. The subject space obtained by INDSCAL. Each point represents the weights assigned by the respective subject to the two axes of the common space (Fig. 4). 
show, in addition, that a logarithmic spacing provides a reasonable approximation to the data of most $S s$. Moreover, the estimated weights for individual $S s$ in Fig. 5 are consistent with the results of previous analyses. For example, all three $S s(5,14,16)$ for which the estimated weights for the vertical dimension far exceed those for the horizontal dimension were $S s$ for which $S$ was dominated by $A$. Similarly, the three $S s(3,4,8)$ whose weights exhibit the opposite pattern were $S s$ for which $A$ was dominated by $S$ (see Table 10).

However, the multidimensional-scaling analysis, summarized in Figs. 4 and 5, fails to represent some highly consistent and significant patterns uncovered in earlier analyses. Such patterns include the increase in the dissimilarity of a given $A$ interval as $S$ becomes more extreme (Table 3), and the relative straightness of $A$ when compared to other dimensions (Table 11). Moreover, some of the observed individual differences, e.g., Table 10, cannot be captured merely in terms of differential weights.

\section{Discussion}

In the present study we investigated two conditions (interdimensional additivity and intradimensional subtractivity) that were proposed as defining properties of subjective dimensions. The purpose of the study was both methodological and substantive. The present paper showed in detail how additivity and subtractivity can be tested, for specified dimensions, on the basis of ordinal dissimilarity data. It also provided information about the manner in which subjects integrate physical dimensions in a simple perceptual task. The major result of this study is the systematic rejection of the tested properties, by phenomena that suggest alternative rules for combining dimensions.

The main reason for the failure of decomposability (Eq. (3)) is the tendency for an interval along one dimension to appear larger the higher or more extreme the level of the orthogonal dimension. The most pronounced effect of this type, in the present study, is the increased dissimilarity for a given pair of shapes as the (equal) area value increases. The reader can easily observe this effect by comparing the dissimilarity between rectangles 1 and 3 to that between 7 and 5 (see Fig. 1). The analogous increase in dissimilarity produced by a given area interval, as shape becomes more extreme, is smaller but nevertheless significant. The latter effect was also reported by Wender (1971). The data, therefore, reject the hypothesis that area and shape contribute independently to the overall similarity between rectangles. They also show that the interaction between the dimensions is very orderly and readily interpretable.

Interaction between perceptual dimensions is probably a widespread phenomenon that may take different forms. The experiments on rectangles reveal an augmentation effect: the perceived difference along one dimension is augmented as the (constant) value of the other dimension increases. Augmentation may hold for many pairs of well-integrated dimensions. Hue differences, for example, are likely to become 
more pronounced as saturation increases. For other pairs of dimensions, one may obtain a reduction effect: the perceived difference along one dimension is reduced as the (constant) value of the other dimension increases. For example, suppose each stimulus consisted of a square plus a rectangle; then the dissimilarity produced by squares of different area would probably be reduced if the (common) rectangles were enlarged, or became more extreme in shape.

These effects suggest new qualitative principles. Suppose $\delta\left(a s, a s^{\prime}\right) \geqslant \delta\left(a^{\prime} s, a^{\prime} s^{\prime}\right)$. That is, the shape interval $\left(s, s^{\prime}\right)$ appears larger at area level $a$ than at area level $a^{\prime}$. One may be tempted to conclude from this observation that $a$ augments shape differences more than $a^{\prime}$. For this conclusion to be valid, however, the augmentation ordering of area levels must be independent of the selected shape interval. Stated formally,

$$
\delta\left(a s, a s^{\prime}\right) \geqslant \delta\left(a^{\prime} s, a^{\prime} s^{\prime}\right) \quad \text { iff } \quad \delta\left(a s^{\prime \prime}, a s^{\prime \prime \prime}\right) \geqslant \delta\left(a^{\prime} s^{\prime \prime}, a^{\prime} s^{\prime \prime \prime}\right)
$$

This condition states that if one shape interval, say $\left(s, s^{\prime}\right)$, appears larger at level $a$ than at level $a^{\prime}$ then any shape interval, say $\left(s^{\prime \prime}, s^{\prime \prime \prime}\right)$, must appear larger at level $a$ than at level $a^{\prime}$. This is the key ordinal property that is necessary for a consistent augmentation (or reduction) model. Note that, under decomposability, Eq. (4) holds because both inequalities reduce to equalities.

The results of the present study also suggest the following ordinal property. If $a^{\prime}$ is between $a^{\prime \prime}$ and $a$ in area, i.e., $a\left|a^{\prime}\right| a^{\prime \prime}$, then

$$
\delta\left(a s, a s^{\prime}\right) \geqslant \delta\left(a^{\prime} s, a^{\prime} s^{\prime}\right) \quad \text { iff } \quad \delta\left(a^{\prime} s, a^{\prime} s^{\prime}\right) \geqslant \delta\left(a^{\prime \prime} s, a^{\prime \prime} s^{\prime}\right) .
$$

This equation asserts that the augmentation ordering of area levels (relative to shape), must coincide with the natural (betweenness) ordering of the levels. This property, which emerged from the results of the present experiment, permits only two types of interactions: augmentation and reduction. (Note that, under decomposability, all four dissimilarities in Eq. (5) should be equal.)

Perhaps the simplest functional form that allows for interaction between dimensions and satisfies Eqs. (4) and (5) is the following model. Let $D\left(a, a^{\prime}\right)=\left[f(a)-f\left(a^{\prime}\right)\right]^{2}$, $D\left(s, s^{\prime}\right)=\left[g(s)-g\left(s^{\prime}\right)\right]^{2}, M\left(a, a^{\prime}\right)=\left[f(a)+f\left(a^{\prime}\right)\right] / 2$ and $M\left(s, s^{\prime}\right)=\left[g(s)+g\left(s^{\prime}\right)\right] / 2$ where $f$ and $g$ are, respectively, the subjective scales for area and shape. Using this notation we write

$$
\begin{aligned}
\delta\left(a s, a^{\prime} s^{\prime}\right) & =F\left[D\left(a, a^{\prime}\right)+D\left(s, s^{\prime}\right)+\alpha M\left(a, a^{\prime}\right) D\left(s, s^{\prime}\right)+\beta M\left(s, s^{\prime}\right) D\left(a, a^{\prime}\right)\right] \\
& =F\left[D\left(a, a^{\prime}\right)\left(1+\beta M\left(s, s^{\prime}\right)\right)+D\left(s, s^{\prime}\right)\left(1+\alpha M\left(a, a^{\prime}\right)\right)\right] .
\end{aligned}
$$

The value of $M$ can be viewed as the location parameter of the respective interval. The parameters $\alpha$ and $\beta$ determine the direction and the magnitude of the interaction between the dimensions. For positive values of $\alpha$ and $\beta$, Eq. (6) produces augmentation; for negative values of $\alpha$ and $\beta$ it produces reduction. Note that $\alpha$ and $\beta$ are independent of each other; area can augment shape while shape may have no effect 
on area. The above model satisfies decomposability if and only if both $\alpha$ and $\beta$ vanish, in which case Eq. (6) reduces to Euclidean form. Furthermore, it satisfies intradimensional subtractivity for any set of stimuli that vary on one dimension only. It should also be noted that while the functional form introduced in Eq. (6) is more complicated than the previous ones, it introduces only two new parameters.

The presence of interactions between attributes suggests two alternative research strategies: (i) to regard natural attributes (such as area and shape) as psychological dimensions, and replace Eqs. (1) and (2) by more complicated rules such as Eq. (6); (ii) to maintain additivity and subtractivity as essential properties of psychological dimensions, and search for factors that satisfy them. The first approach calls for the formulation, analysis, and testing of interactive rules for combining dimensions of the type illustrated in Eq. (6.) The second approach is adopted, in effect, in most models for multidimensional scaling which assume both additivity and subtractivity in the computation of distance. The presence of interactions between attributes is reflected, in these models, by the nonparallelism of lines consisting of points that vary on one attribute only (see Fig. 4), and by the introduction of additional dimensions.

The choice between the two approaches depends, in part at least, on the purpose of the investigation. If one is concerned with data reduction and convenient display of results, the latter approach has somc advantages. If, on the other hand, onc is primarily concerned with the manner in which people perceive and integrate stimuli, the former approach seems more appealing.

Throughout this paper the emphasis has been on the detection of systematic departures from qualitative principles, rather than on overall measures of goodnessof-fit. This approach led to the rejection of a large class of multidimensional scaling models, and suggested a new class of models for future investigation.

\section{REFERENCES}

Beals, R., Krantz, D. H., \& Tversky, A. Foundations of multidimensional scaling. Psychological Review, 1968, 75, 127-142.

Carroll, J. D., \& Chang, J. J. Analysis of individual differences in multidimensional scaling via an $N$-way generalization of "Eckart-Young" decomposition. Psychometrika, 1970, 35, 283-319.

Coombs, C. H. $A$ theory of data. New York: Wiley, 1964.

KLAHR, D. A Monte Carlo investigation of the statistical significance of Kruskal's nonmetric scaling procedure. Psychometrika, 1969, 34, 319-333.

Krantz, D. H., Luce, R. D., Suppes, P., \& Tversky, A. Foundations of measurement. Vol. I. New York: Academic Press, 1971.

Kruskal, J. B. Multidimensional scaling by optimizing goodness of fit to a nonmetric hypothesis. Psychometrika, 1964, 29, 251-263.

SuPpes, P., \& WINET, M. An axiomatization of utility based on the notion of utility differences. Management Science, 1955, 1, 259-270. 
Tversky, A., \& Krantz, D. H. Similarity of schematic faces: A test of interdimensional additivity. Perception \& Psychophysics, 1969, 5, 124-128.

Tversky, A., \& KRANTz, D. H. The dimensional representation and the metric structure of similarity data. Journal of Mathematical Psychology, 1970, 7, 572-596.

Wender, K. A test of independence of dimensions in multidimensional scaling. Perception $\xi$ Psychophysics, 1971, 10, 30-32.

RECEIVED: October 2, 1973 